\title{
Retraction Note to: Triptolide inhibits angiogenesis in microvascular endothelial cells through regulation of miR-92a
}

\author{
Xiaomeng Xu ${ }^{1,2} \cdot$ Li Tian $^{3} \cdot$ Zhimian Zhang $^{1}$ (1) \\ Published online: 14 February 2022 \\ (c) The Author(s) under exclusive licence to University of Navarra 2022
}

\section{Retraction Note to: Journal of Physiology and Biochemistry (2019) 75:573-583 https://doi.org/10.1007/s13105-019-00707-2}

The Editors-in-Chief have retracted this article following an investigation by the Chinese Ministry of Science and Technology. The investigation found the data presented here has been either forged or tampered with. The Editors-in-Chief therefore no longer have confidence in the integrity of the data in this article.

None of the authors have responded to any correspondence from the Editor or Publisher about this retraction.

Publisher's note Springer Nature remains neutral with regard to jurisdictional claims in published maps and institutional affiliations.

The original article can be found online at https://doi.org/10.1007/ s13105-019-00707-2

Zhimian Zhang

zhangzhimian0064@sina.com

1 Medical Examination Center of Qilu Hospital of Shandong University, No.107 Culture West Road, Jinan 250012, Shandong, China

2 Department of Health Management, Jining NO.1 People's Hospital, Jining 272011, Shandong, China

3 Department of Critical Care Medicine, Jining NO.1 People's Hospital, Jining 272011, Shandong, China 